\title{
VIOLATING THE BASIC TENETS OF CARE - LOOKING BACK ON YEARS OF EVIDENCE BASED MEDICINE FROM AN ONCOLOGIST'S VIEWPOINT
}

\section{Dr. Bishan Basu*}

Associate Professor \& Head, Department of Radiotherapy, Calcutta National Medical College, 32 Gorachand Road, Kolkata 700014. ${ }^{*}$ Corresponding Author

ABSTRACT Evidence-Based Medicine (EBM) came into fashion nearly three decades ago. However, over this short period, this system of medical philosophy has come to dominate the medical practice worldwide. Never in the history of medicine could a single way of medical practice could dominate the healthcare systems of diverse countries like EBM did, that too within such short time span. But, it is high time we should ponder over the pros and cons of EBM and if this way of medical practice is to be allowed to continue, we should consider integration of additional inputs from the traditional ways of medicine. Though article focusses upon the cancer care, the conclusions derived can be applied to any other disciplines of healthcare.

\section{KEYWORDS :}

Nearly three decades have elapsed since the Evidence-Based Medicine Working Group came up with the basic concepts and premises of Evidence-Based Medicine (EBM) (1).

To provide some kind of philosophical background for the move towards EBM from the traditional authoritative medical practice, the Working Group had used Thomas Kuhn's (2) concepts of the scientific revolution and his phrase 'paradigm shift'.

While numerous scholars have argued that Kuhn's concepts of scientific revolution and paradigm shift are for the physical sciences only and should not be used to interpret the progress of medical sciences, I, personally, find the Working Group's use of those terms justified. However, though the Working Group tried to explain that the revolution, and the accompanying shift in paradigm, had occurred in the medical science itself through the popularity of randomized controlled trials (RCT), I think that the true paradigm shift had occurred in the economics of medical research and healthcare that demanded a shift of medical thought process from traditional way of caring for patients to EBM.

Veatch et al described Kuhn's basic hypothesis in a succinct way (3). To quote from their article, "Kuhn takes the practice of science to consist in relatively long periods of 'normal science,' which occasionally are interrupted by 'scientific revolution.' Following a scientific revolution, there ensues a new period of normal science. Normal science is characterized by a 'paradigm,' an accepted way of proceeding in the asking of questions and seeking answers to questions. Normal science does not aim to produce major novelties. It is more of an exercise of puzzle-solving. A paradigm delineates the sorts of questions that may be asked by scientists, limits the nature of acceptable answers to these questions, and specifies the methods that may be used in the process. Paradigms are adopted by particular scientific communities and need not be shared by all scientists. During a period of normal science, the current paradigm is successful both in generating a rich supply of puzzles to be solved and in formulating answers to these puzzles. As long as this is the case, a paradigm is relatively stable. However, eventually an anomaly, a puzzle that cannot be solved within the paradigm, will arise. The appearance of a significant anomaly is a 'crisis.' Such crises loosen the rules of the normal way of proceeding under the paradigm. Ultimately a new paradigm is found that accommodates the anomaly and goes on generating new puzzles and solu-tions. This paradigm replacement is a 'scientific revolution."' methodology of medical research - particularly in the aspects of how it is done, why it is done, where it is done and most importantly, how such research activities are funded. In this new paradigm of corporate profit-driven enterprise of medical research, EBM has come up as the most effective and efficient puzzle-solver.

Another one of Kuhn's key concepts - the concept of 'inco mmensurability' (2) between the pre- and post-revolutionary scientific paradigms, a concept first propounded by Paul Feyerabend (4) - is also applicable here. Kuhn noted, proponents of the competing paradigms fail to make contact with others views as there is seldom any continuity between the two very different paradigms - the pre- and postrevolutionary scientific ideas are incommensurable to each other. To put this theory in the context of medicine, medical research - and the way evidence are generated from such activities - at its present avatar, is barely explainable from the traditional medical value system, since two systems belong to very different paradigms. Veatch et al in their previously mentioned article enumerated the stages of clinical decision making this incommensurability may affect (3)

In this era of financial profit-driven medical research, instead of finding effective cure for existing life-threatening diseases, a major part of research activities is spent on finding out illnesses to develop and promote the newer expensive drugs a concept barely understandable from traditional medicine's point of view - so much so that we must appreciate the foresight of Ivan Illich (5) : "The medical establishment has become a major threat to health". Even when the life-threatening diseases are addressed, focus is on the chronic treatment instead of finding a cure-because, that is where the money is. When those new drugs comes into market, they get their approval without any "evidence" of proper benefit - at least not the way benefit was defined by the previous generation of medicine. For example, analysis of new cancer medicines approved by the European Medicines Agency between the year 2009 and 2013 revealed that most anti-cancer drugs approved within that time span got their approval without evidence of benefit on survival or improvement in quality of life. And when the results of those medicines were followed up over some three or four years after coming into market, their benefits were still doubtful. (6) Without the dogma of EBM, such research activities could hardly succeed. And drug companies, particularly in oncology, are trying hard to find newer and novel endpoints for their clinical trials - endpoints which might earn them a quick approval, but hardly relevant in clinical settings. 
talking about? As we all know, there is a hierarchy of evidence, as per EBM guidelines. Mechanistic reasoning e.g. pathophysiologic rationale is not encouraged by EBM. But, when the newer technologies are readily incorporated in routine practice and $\mathrm{RCT}$ protocols - without sufficient evidence in favour of it - should not that count as mechanistic reasoning? Afterwards, use of newer technology becomes commonplace and such use gets almost post facto validity. In the field of radiation oncology, newer technology are coming out at quite a frequent interval and almost every newer technology is touted as better than the previous one. In her book titled 'Cancer, Radiation Therapy and the Market', Barbara Bridgman Perkins had argued quite convincingly that the recent growth of radiation therapy technology had more to do with the device industry market than with the elusive cure of cancer. (7) And the USFDA's 510(k) clearance process made things easier for the device manufacturers. Through this clause, a new technology may be allowed to come into healthcare market merely by comparing the newer one with a previously licensed similar technology, thereby removing the requirements for fresh evaluation of new devices during the licensing process.

New machines and new technologies always get more attention than the previous ones and even before sufficient evidence is gathered, adoption of new technology becomes the 'new normal'. Even in the early days of IMRT (an advanced technology of radiation therapy), the technology was quickly adopted without waiting for sufficient positive evidence in favour of it. Initially, the new technology was used to treat highrisk patients (where results with older technology might have been unsatisfactory), but adoption of the new technology quickly spread across the board, thereby hugely expanding treatment costs. (8) Evidence in favour of IMRT in the treatment of select malignancies came later, but the hype and popularity in favour of the new technology preceded such evidence and that popularity, most definitely, helped to gather those evidences.

This hype, sometimes backed by mainstream media, about newer and expensive machines is incongruous with the healthcare reality of the nation. So, while in terms of availability of radiation therapy facilities per million population India is comparable to poorest countries in the sub-Saharan Africa (9), and while we as a nation may be having the largest number of people below the poverty line (10), that does not prevent us from opting for Rs. 500 crore plus proton beam therapy machines, the treatment might cost more than 25 lakhs per patient (11).

All these are happening while evidence in favour of proton therapy are minimal. While the biology and radiation dose distribution with proton therapy is encouraging, the dose distribution does not always result in better clinical outcome and anyway, evidence-based medicine does not care for such mechanistic or logical reasoning. However, in a recent metaanalysis compiling all proton therapy trials, the authors found that out of 219 studies only $41 \%$ were prosoective ones and very few of them were randomized. Research designs and/or findings were inadequately reported in as many as as $83 \%$ of such prospective studies. The authors concluded that prospective randomized evidence, supposedly the gold standard in evidence based medicine, in favour of proton beam therapy is limited. (12). History has taught us that with such big investments and such marketing hypes, favourable evidence would surely follow in due time. To put it in another way, if a new technology is there, can favourable evidence be far away?

Almost in every discipline, nearly every new drug is compared to placebo and hardly ever the new one get compared with an existing older one. Let us take the example of oncology again. In the arena of oncology medicines, the scenario has deteriorated to such low levels that the USFDA had to come forward with a detailed guideline for the drug industry to systematize and restrict the use of placebos in the cancer drug development trials. (13)

In the rare occurrences when the new drugs are compared to an existing effective therapy, the design of the clinical trial is invariably a 'non-inferiority' one. Results from RCTs with positive findings are published with much advertising, whereas the trials with negative results are rarely published. Even, the results of 'non-inferiority'-designed RCTs are misrepresented to make the new drug look superior. So, in the basic process of generating the evidence, the balance is tilted towards any new drug whatsoever. Two decades ago, Bodenheimer noted that the major financial burden of new drug development was borne by the pharmaceutical industry and he also observed some hindrances when authors went on to publish negative or unsatisfactory results. (14) The situation has worsened. Delgado et al in their meta-analysis of clinical trials, results of which were published in three major journals between 2013 and 2015, found that the randomized controlled trials which were funded by companies with commercial interests were more likely to show favourable outcome and this difference is mainly due to unusual surrogate endpoints adopted by the for-profit funded clinical trials. (15) So, even before starting to put the evidence into concrete practice, the 'evidence' itself is hardly above question.

While there is distinct bias towards generating evidence in favour of newer therapies, further problem arises in adopting that evidence to treat individual patients. As an inevitable corollary to the basic tenets of EBM, ethnic diversities are ignored to treat all human beings, throughout the world, as a monolithic community - otherwise, one could hardly be able to incorporate the so called level I evidence generated from one $\mathrm{RCT}$ to treat an individual patient residing at the other end of globe. Furthermore, statistical data obtained from a group of patients can be extrapolated to another similar group of patients, but application of this same knowledge to an individual patient is problematic. (16) Data obtained from experimenting upon a group is of course a good evidence to act upon to another equally matched group, but what happens when the groups are not matched. But, who cares?

Therefore, in this new paradigm of corporate profit-driven medical research, EBM has become an excellent puzzlesolver by helping a specific kind of research activity gather evidence in favour of all kinds of newer technologies and therapies and by promoting them effectively. EBM has become so successful in this endeavour that the state-funded healthcare system of the developing countries have reached the level of near bankruptcy - and the developed nations are not falling behind either - by chasing the newer, expensive but, not necessarily superior - technologies and therapies.

This statement needs further clarification, because it has been argued that evidence-based medicine can be an effective tool in bringing down the spiralling cost of healthcare by limiting the number of unnecessary and futile tests or procedures etc. Lets go by the example of cancer again. Neubauer et al concluded from their study that treating patients according to evidence-based guidelines is a cost-effective strategy for delivering care to those with non-small cell lung cancer, and following evidence-based approach can, in fact, reduce cost (17). However, a more detailed analysis reveals a completely different picture. Warren et al had done a detailed analysis of evolution of costs of treatment for four major cancers over one decade (1991 - 2002). In their analysis, they included only the initial costs of treatment, as per their definition costs occurring from 2 months before diagnosis to 12 months following diagnosis. Predictably, they found a significantly increase in costs for the treatment of cancer, particularly so in the treatment of lung and colorectal cancer. Only exception was 
prostate cancer, where treatmrnt cost actually decreased, perhaps due to the reduction in the number of surgeical intervention done for that disease (18). And let us not forget a few important facts while going through the results of this study. The period of study ended in 2002, before the surge of super-expensive targeted therapy drugs. Also, lots of expensive radiation therapy technology were not widely available then. Secondly, though treatment of advanced cancer is a prolonged process (and with discovery of every new treatment, it is getting more and more prolonged), the study included only the costs for the first year. Thirdly, majority of the expensive newer drugs are targeted towards the advanced stage of the disease and with the goal of cancer therapy changing to stabilize the disease rather than to cure it, treatment is more prolonged than ever. So, if we relate all these factors with the data provided by these article, the true cost has really skyrocketed. And if we try to interpret these findings in terms of the Indian scenario and consider the almost inevitable increase in incidence of cancer in our country, the fragile state-supported healthcare programme can hardly bear such expenses.

Therefore, while EBM has been spectacularly successful in inflating the profits of corporate health industry, there are plenty of data to display that this constant drive to chase the latest expensive technologies and therapies - an inevitable adverse outcome of EBM, particularly in its present form - has proven to be disastrous for the state-supported healthcare systems throughout the world. Still, it might be too early to refute EBM, particularly its basic principles, though its socioeconomic consequences are all too clear. Therefore, in addition to a thorough knowledge in medical statistics, as touted by the proponents of EBM, individual physicians should better travel back in time, take some valuable inputs from the great Hippocrates and Galen, gather some training in rationality, logic, ethics and be socially responsible. That should be the only way forward.

Alfred Worcester said almost a century ago: "The younger members of the profession, although having enormously greater knowledge of the science of medicine, have less acquaintance than many of their elders with the art of medical practice..... Primarily, it (i.e. the art of medical practice) depends upon devotion to the patient rather than to his disease." (19) In its present avatar, EBM encourages more to focus upon the ailment an individual is suffering from than the individual human being. A generation of physicians taught on these principles might be more beneficial for the healthcare industry than the individual patients. If EBM is allowed to reign, more attention needs to be paid on the source of funding behind the evidence, why and how should that evidence be interpreted before applying such for the care of an individual patient and when should the physician look beyond the evidence to care for an individual human being.

REFERENCES :

1) Evidence-Based Medicine Working Group. Evidence-based medicine. Anew approach to teaching the practice of medicine. JAMA. 1992 Nov 4;268(17):2420-5

PubMed PMID: 1404801

2) Kuhn TS : The Structure of Scientific Revolutions, Chicago, Ill; University of Chicago Press, 1970

3) Veatch RM, Stempsey WE. Incommensurability: its implications for the patient/physician relation. J Med Philos. 1995;20(3):253-269. doi:10.1093/jmp /20.3.253

4) Feyerabend P: "Explanation, Reduction and Empiricism", in H. Feigl and G. Maxwell (ed.), Scientific Explanation, Space, and Time, (Minnesota Studies in the Philosophy of Science, Volume III), Minneapolis: University of Minneapolis Press, 1962

5) Illich, Ivan. Medical Nemesis; the Expropriation of Health; New York; Pantheon Books, 1976

6) Davis C, Naci H, Gurpinar E, Poplavska E, Pinto A, Aggarwal A. Availability of evidence of benefits on overall survival and quality of life of cancer drugs approved by European Medicines Agency: retrospective cohort study of drug approvals 2009-13. BMJ. 2017;359:j4530. Published 2017 Oct 4. d o i: 10.1136 /bmj.j4530

7) Perkins, Barbara Bridgman : Cancer, Radiation Therapy and the Market ; Taylor and Francis ; 2019

8) Jacobs BL, Zhang Y, Skolarus TA, Hollenbeck BK. Growth of high-cost intensity-modulated radiotherapy for prostate cancer raises concerns about overuse. Health Aff (Millwood). 2012;31(4):750-759. doi:10.1377/hlthaff. 2011 1062

9.) IAEA Availability of radiotherapy. Number of radiotherapy machine per million persons. IAEA; 2012. Available from: http://www.iaea.org/newscente /focus/cancercare/dirac2012.pdf. [Last accessed on 03/08/2020)

10) The World Bank. Poverty \& Equity. India. The World Bank; 2013. Available from: http://povertydata.worldbank.org/poverty/country/IND.

11) https://www.thehindu.com/news/cities/chennai/apollo-hospitals-inaug urates-proton-cancer-centre/article26083995.ece (accessed on 03/08/2020)

12) Ofuya M, McParland L, Murray L, Brown S, Sebag-Montefiore D, Hall E. Systematic review of methodology used in clinical studies evaluating the benefits of proton beam therapy. Clin Transl Radiat Oncol. 2019 Jul 12;19:1726. doi: 10.1016/j.ctro.2019.07.002. PMID: 31372521; PMCID: PMC6660607.

13) https://www.fda.gov/regulatory-information/search-fda-guidancedocuments/placebos-and-blinding-randomized-controlled-cancer-clinicaltrials-drug-and-biological-products accessed on 12/07/2020

14) Bodenheimer T. Uneasy alliance--clinical investigators and the pharmaceutical industry. N Engl J Med. 2000;342(20):1539-1544. doi:10.1056 /NEJM200005183422024

15) Falk Delgado A, Falk Delgado $A$. The association of funding source on effect size in randomized controlled trials: 2013-2015 - a cross-sectional survey and meta-analysis. Trials. 2017;18(1):125. Published 2017 Mar 14. doi:10.1186/s 3063-017-1872-0

16) Tonelli MR. The philosophical limits of evidence-based medicine. Acad Med. 1998:73(12):1234-1240. doi:10.1097/00001888-199812000-00011

17) Neubauer MA, Hoverman JR, Kolodziej M, Reisman L, Gruschkus SK, Hoang S, Alva AA, McArthur M, Forsyth M, Rothermel T, Beveridge RA. Cost effectiveness of evidence-based treatment guidelines for the treatment of non-small-cell lung cancer in the community setting. J Oncol Pract. 2010 Jan;6(1):12-8. doi: 10.1200/JOP.091058. Epub 2009 Dec 30. PMID: 20539725; PMCID: PMC2805337.

18) Warren JL, Yabroff KR, Meekins A, Topor M, Lamont EB, Brown ML. Evaluation of trends in the cost of initial cancer treatment. J Natl Cancer Inst. 2008 Jun 18;100(12):888-97. doi: 10.1093/jnci/djn175. Epub 2008 Jun 10. PMID: 18544740; PMCID: PMC3298963.

19) Worcester, A.The care of the aged, the dying and the dead (1935), Baltimore \& Co, London 